\title{
Approche négociée de formation d'équipes éducatives : des conditions de réussite
}

Yannick Bruxelle et Dominique Ellinger

\section{OpenEdition}

Édition électronique

URL : http://journals.openedition.org/ere/6579

DOI : $10.4000 /$ ere. 6579

ISSN : 2561-2271

Éditeur

Centr'ERE

Référence électronique

Yannick Bruxelle et Dominique Ellinger, «Approche négociée de formation d'équipes éducatives : des conditions de réussite », Éducation relative à l'environnement [En ligne], Volume 3 | 2002, mis en ligne le 20 mars 2002, consulté le 16 avril 2021. URL : http://journals.openedition.org/ere/6579 ; DOI : https:// doi.org/10.4000/ere.6579

Ce document a été généré automatiquement le 16 avril 2021. 


\title{
Approche négociée de formation d'équipes éducatives : des conditions de réussite
}

\author{
Yannick Bruxelle et Dominique Ellinger
}

1 Dans l'institution Éducation nationale française, la formation continue des enseignants et des personnels du second degré (élèves de 11 à 19 ans) relève de deux logiques et de deux dynamiques complémentaires : celle de «l'offre » venant de l'institution sous la forme de catalogues annuels de propositions appelés « plans de formation » permettant aux enseignants et non-enseignants de poser leur candidature pour participer aux stages qui les attirent, et celle de «la demande » émanant des établissements euxmêmes en fonction de leurs besoins spécifiques.

2 Les demandes des établissements dans le domaine de l'éducation relative à l'environnement (ERE) sont récentes et encore peu nombreuses dans l'académie de Poitiers. Ainsi, deux demandes ont été reçues cette année scolaire par l'IUFM (Institut Universitaire de Formation des Maîtres) et transmises à la DAAC (Délégation Académique à l'Action Culturelle, anciennement MAAC - Mission Académique à l'Action Culturelle) du Rectorat qui a en charge l'éducation à l'environnement dans son approche culturelle interdisciplinaire. Les thématiques demandées concernaient d'une part la découverte plurielle (historique, économique, géographique, naturaliste, etc.) d'un territoire rural (lycée $\mathrm{A}$ ) et d'autre part l'analyse d'un problème complexe lié à la construction récente d'un barrage (lycée B).

3 Notre réponse comme pour tous les autres stages s'est inscrite dans le cadre d'une part d'un partenariat institutionnel, ici entre l'IUFM, la DAAC et des CPIE (Centres Permanents d'Initiatives pour l'Environnement) proches du lieu de l'établissement et d'autre part d'une approche interdisciplinaire.

4 Nous sommes toutes les deux formatrices de l'Éducation nationale dans le domaine de l'éducation à l'environnement et appartenons à une équipe partenariale plus large, habituée à travailler ensemble depuis plusieurs années. Ces demandes de formation relativement nouvelles pour nous, largement plus complexes à gérer, mais aussi nos 
habitudes de travail dans une équipe en recherche-action, nous ont stimulées et entraînées à mener cette petite réflexion sur les pratiques professionnelles liées à ce type de stages.

5 En tant que praticiennes, nous avons souhaité rendre visible cette expérience, apporter quelques indices sur les qualités, intérêts et limites de ce dispositif et si possible proposer quelques pistes d'améliorations.

6 Nos hypothèses de départ étaient :

- que ces stages menés en concertation formateurs-stagiaires, de la préparation jusqu'au suivi, étaient mieux adaptés au terrain que d'autres formations ;

- que notre pratique de formateurs en équipe partenariale renforçait la notion d'équipe éducative dans les établissements scolaires et ainsi favorisait l'approche nécessairement interdisciplinaire de l'ERE;

- que ces stages seraient donc globalement pertinents et efficaces au niveau des transferts avec les élèves ;

- qu'ils seraient de plus efficients pour l'institution, car moins coûteux financièrement (théoriquement aucun frais de déplacements, ni de repas).

7 Pour essayer de développer et de clarifier cette hypothèse, nous nous appuierons sur la description des spécificités de ces stages à partir de l'expérience vécue avec deux lycées. Nous prendrons en compte les analyses des indices de satisfaction "à chaud » des stagiaires et les résultats de questionnaires individuels adressés aux stagiaires respectivement dix mois (lycée $A)$ et six mois (lycée B) après la formation $(20 \%$ de retours en tout $)^{1}$

\section{Une démarche dans la durée}

\section{Une rencontre préalable entre les formateurs et l'équipe de l'établissement, plusieurs semaines voire plusieurs mois avant le stage}

8 De façon générale, les formulaires de demandes de formation qui nous parviennent restent souvent brefs, vagues et surtout, nous ne savons jamais par qui ils ont été rédigés (sont-ils ou non l'objet d'une concertation préalable ?). Un premier contact téléphonique avec le chef d'établissement peut déjà apporter quelques précisions, mais aussi nous permet d'éviter tout malentendu ultérieur : notre approche sera forcément interdisciplinaire et fera appel à des partenaires extérieurs à l'Éducation nationale (si la demande était spécifiquement monodisciplinaire, elle serait transmise aux inspecteurs pédagogiques régionaux de la discipline). Il permet aussi d'expliquer la nécessité d'une rencontre préalable avec les personnes concernées pour affiner, cadrer la demande et ébaucher ensemble les premières propositions.

9 La démarche alors mise en œuvre pour faire préciser la demande de stage par l'équipe de l'établissement s'inspire de la méthode classique dite de Quintillien (qui ? quoi ? où? quand ? comment? pourquoi ? combien?).

10 Ainsi dans le lycée $A$, il s'avère que la demande est surtout portée par le chef d'établissement à la convergence d'une attente des professeurs de sciences économiques et du départ à la retraite d'un enseignant de sciences de la vie et de la terre, sans forcément s'appuyer sur une véritable démarche interdisciplinaire au 
départ : nous n'étions pas assez nombreux, alors nous avons réuni des objectifs de formation en économie et en écologie, par contre elle associe des personnels enseignants de plusieurs disciplines et des non-enseignants (agents, gestionnaires, etc.). Dans le lycée B, la demande émane d'équipes pédagogiques pluridisciplinaires (une dizaine de disciplines différentes) déjà actives et réflexives depuis des années dans l'établissement.

11 La demande porte, pour le lycée A, sur une meilleure connaissance du lieu: besoin de mieux connaître l'environnement économique pour aider les élèves à s'insérer dans la vie active ou à trouver des terrains de stages, alors que la demande du lycée B est plus pédagogique et méthodologique : pour que l'ensemble des équipes se retrouvent pour échanger et améliorer leur fonctionnement, pour répondre au problème de la mise en cuvre d'un projet et pour améliorer l'efficacité des sorties avec les élèves.

Il y avait un décalage certain entre leur vision de l'environnement et ce que nous pouvions et souhaitions leur apporter d'où la nécessité lors de la concertation de trouver des convergences pour avancer vers l'ERE.

Dans le lycée $\mathrm{B}$, le choix du sujet est affiné de façon à pouvoir être déclinable à diverses situations en fonction des équipes pédagogiques et devient "approche globale et citoyenne d'un sujet polémique " à partir de l'exemple d'un barrage (le terme de " polémique " posera d'ailleurs problème et sera par la suite abandonné à la demande des partenaires locaux qui sont au cœur de tensions politiques).

Le lieu précis du stage est aussi décidé lors de la concertation :

- Pour le lycée A : sur place, au lycée même afin d'être le point de départ de travaux sur le terrain.

- Pour le lycée $\mathrm{B}$ : dans le centre du CPIE partenaire, pour deux jours. Ceci répondait à la demande même des équipes de se retrouver ensemble et hors de leur lycée.

Dans les deux cas, les personnes étaient volontaires pour participer à cette formation, mais à des degrés d'investissement divers.

Ces simples exemples peuvent donner une idée de la diversité de demandes, qui, si elles n'étaient pas clarifiées au préalable, nous amèneraient à y répondre d'une façon standardisée et forcément inadaptée.

Il s'agit d'une recherche de partenariat entre l'équipe de l'établissement et l'équipe de formateurs pour qu'ensuite nous devenions coauteurs et coacteurs de cette formation.

Ceci suppose comme nous le verrons plus loin une certaine "posture" positive de la part des uns et des autres nécessitant l'écoute et la prise en compte de points de vue ce qui n'est pas forcément le cas au tout début.

Ainsi dans le lycée A on attendait de notre équipe formatrice qu'elle apporte tout, fasse des recherches locales, en fait tout ce qu'ils n'avaient pas le temps de faire par euxmêmes. Dans le lycée B le premier contact fut méfiant (qui sont-ils? que peuvent-ils bien nous apporter que nous ne sachions déjà ?, etc.), mais dans les deux cas un climat de confiance a pu s'établir et déboucher à l'issue d'un dialogue sur un contrat clair avec un cadre de propositions précises proposées et écrites par les formateurs et validées par les stagiaires.

20 A posteriori cette phase de préparation a été jugée positive par les stagiaires : ces stages répondent vraiment à nos souhaits, car ils sont mis en œuvre après une concertation préalable. 


\section{Un travail de préparation entre formateurs partenaires}

21 Suite à cette première rencontre qui a fixé le cadre du stage, l'équipe de conception va s'étoffer en faisant appel aux personnes semblant le mieux convenir pour la demande : il est donc nécessaire de disposer d'un réseau important de partenaires et d'intervenants ainsi que de lieux ressources. La DAAC, dont la mise en œuvre des partenariats est l'une des missions, est en capacité de répondre à ces demandes en s'entourant des compétences adaptées.

Dans les deux cas, l'équipe de formateurs ainsi constituée nous associait un ou plusieurs responsable(s) pédagogique(s) d'un CPIE, et pour le lycée $\mathrm{B}$, il a été possible d'intégrer un représentant de cet établissement. Pour le lycée A, nous avons sollicité les compétences d'un des stagiaires pour animer l'une des séquences de terrain du stage. Ainsi nous avons fait le choix de développer toutes les possibilités de coopération avec les membres de l'établissement.

Il s'agit donc bien d'un choix négocié des contenus et faisant appel à des spécialistes du contexte local (universitaires, associations, professionnels, membres de l'établissement, etc.).

\section{Un déroulement de stage spécifique de l'ERE}

Nous avons dans les deux cas mis en œuvre une pédagogie active comparable à celle de tous les stages que nous encadrons dans le domaine de l'ERE : prise en compte des représentations, mise en situation sur le terrain, rencontres d'acteurs exprimant une multiplicité de points de vue (relevant aussi bien des aspects économiques, écologiques, sociaux, culturels ou politiques du sujet), apports plus théoriques ou généraux, mise en système des informations débouchant sur des productions par les stagiaires. Nous ne notons donc pas à ce niveau de différence importante par rapport aux stages «à l'offre ».

25 La pratique des méthodes actives est (comme souvent) diversement appréciée : nous sommes en situation d'acteurs-élèves avec une bonne découverte de la difficulté que peuvent rencontrer les élèves dans une telle production ou encore les représentations du début m'ont semblé nous faire perdre du temps et il aurait été préférable de continuer les rencontres.

L'aspect local est spécifique de ce type de stage, il permet une retombée immédiate des contacts offerts : beaucoup d'informations apportées, je repars avec un carnet d'adresses ; j'ai découvert de nouveaux interlocuteurs, d'autres sources de financements de projets.

Mais, dans le même temps, on a pu noter chez certains stagiaires une réticence à passer de la problématique locale qui les soucie directement à une problématique plus large. Est-ce une "résistance ", une attitude "consommatrice » ou n'est-ce pas plutôt une difficulté à articuler les échelles du local au global? Ainsi, une intervention globalisante a pu être jugée : un peu hors sujet par rapport à l'objectif du stage.

\section{Une réflexion sur des projets concrets pour et avec les élèves}

Selon les cas, il s'agit soit d'affiner et d'enrichir des projets déjà existants (lycée B), soit de chercher à en mettre en œuvre (lycée A), mais on constate toujours le souci d'une meilleure pertinence pour les élèves. La volonté de transférer au plus vite les acquis du 
stage est exprimée et typique de ces stages à la demande, même si des angoisses et des limites sont évoquées : nous, on travaille avec des élèves qui ne sont pas tous volontaires pour ce type d'activités.

Les temps d'échanges et de réflexion sur le projet de l'établissement sont essentiels et font la réelle originalité de ce type de stages. Il est donc indispensable de leur réserver des plages

\section{Des relations plus fortes, une prise de risque pour tous...}

30 Nous notons un climat général très particulier pour ces stages, beaucoup d'écoute, mais aussi des moments d'effervescence, des tensions qui se révèlent, pouvant apporter leur cortège de plaisirs et de douleurs pour les deux équipes en jeu.

\section{Une équipe d'établissement qui vit, qui se construit...}

31 Cette équipe existait parfois avant (lycée B) ou se construit et vit pendant le stage (lycée A) et continuera de vivre après... ( ?).

Au départ le stage reflète un engagement personnel, un désir de nouveau, un besoin, une envie d'essayer de nouvelles pratiques, etc. Dans le lycée B : je crois à la grande richesse d'un travail d'équipe; les gens doivent être volontaires; l'envie de mener des travaux interdisciplinaires; être prêts à discuter; avoir le désir de travailler ensemble, avec d'autres collègues. Dans le lycée A : mieux connaître les matières et les préoccupations des collègues; découvrir les connaissances de chacun ;être investis à plusieurs dans cette démarche.

Pendant le stage des tensions peuvent se révéler. Ainsi pour le lycée B : problème de non écoute des autres par certains stagiaires; relations tendues entre stagiaires parfois bornés et peu citoyens; certaines personnes sont peu enclines à la discussion réfléchie préférant critiquer sans objectivité ou critiquer entre elles.

Par la suite, ces tensions ont pu déboucher sur des ruptures, toujours dans l'établissement B: on a pu voir ce que certains mettent derrière travail d'équipe en interdisciplinarité ; le stage n'a fait que révéler certains clivages; le fonctionnement a été amélioré ou clarifié, ce qui est très positif; l'intervention d'une équipe étrangère au groupe a permis de jouer un rôle de catalyseur ou de déclencheur dans certaines équipes des classes de seconde.

\section{Une équipe de formateurs qui s'engage}

Il ne nous semble pas concevable, compte tenu de la complexité des sujets environnementaux et de l'interdisciplinarité des formations, qu'un formateur isolé, quelle que soit sa compétence, soit en mesure de répondre efficacement à ces demandes.

De plus, nous sommes convaincues que ces formations menées «en " partenariat sont des formations « $a u$ " partenariat et nous pensons que des formations proposées par des équipes de formateurs sont à même d'être des formations au travail en équipe. Il nous semble que c'est de la confrontation de ces équipes (formateurs et stagiaires), de leurs façons de travailler, de s'organiser, de se doter d'outils spécifiques, etc. et de la 
richesse de leurs échanges que peut naître la découverte du plaisir, de l'efficacité de ce type de relations professionnelles. Quant à la découverte de l'impact des pratiques réflexives, il s'agirait d'un autre chantier à explorer.

Tout ceci suppose une équipe qui, dès le départ, accepte de prendre en compte la spécificité de chaque demande, d'innover dans ses pratiques, de prendre des risques face à du « jamais fait » et qui accepte aussi un fort investissement en temps. C'est à ce prix qu'elle découvrira les plaisirs de la «réciprocité éducative » et du «partenariat apprenant » (Bruxelle, 2001), créateurs de savoirs nouveaux.

\section{Des établissements qui évoluent}

Quelques mois se sont écoulés, les démarches mises en œuvre dans les deux établissements ont été différentes.

Dans le lycée A: le chef d'établissement a changé, des professeurs ont quitté l'établissement, les demandes d'adaptation des emplois du temps qui semblaient conditionner la mise en place du projet ébauché au cours du stage n'ont pas été réalisées : l'administration n'a pas suivi. Le questionnaire, dix mois après révèle que : il ne s'est rien passé, le projet était plus personnel que collectif.

Dans le lycée B: nous avons fait un bilan du stage lors de notre concertation; plusieurs collègues ont regretté de ne s'être pas inscrits; nous avons eu envie de mettre en pratique et de nous investir dans des projets similaires; nous avons pu l'appliquer directement en mettant en cuvre notre sortie avec élaboration d'un carnet de terrain et d'un travail de groupe. Quant à l'administration du lycée : elle n'a pas regretté la désorganisation provoquée par le stage.

41 Dans ce lycée, trois projets différents relatifs à l'éducation à l'environnement et à la citoyenneté ont été inscrits au projet d'établissement et financés dans différents cadres partenariaux.

42 À ce jour, des demandes de suivi sont clairement formulées à l'équipe de formation (un regard sur les productions, une aide pour développer de nouveaux projets, le lancement d'un travail d'écriture sur les pratiques, etc.).

\section{Bilan, perspectives et propositions}

\section{Quelle pertinence ? Quelle efficacité ? Quelle efficience ? pour ces stages « à la demande »}

Pertinence : comme nous venons de le voir, les différents éléments recueillis aussi bien dans les évaluations à chaud que dans les évaluations différées nous permettent de dire que ces formations sont effectivement pertinentes pour l'aide à la mise en place de projets interdisciplinaires en ERE qui « collent » à la réalité locale.

Efficacité : l'étude fait apparaître que de nombreux paramètres entrent en ligne de compte, y compris d'ailleurs des éléments extérieurs au stage. Le stage n'est pas isolé du reste, il prend sa place dans un processus plus général et ne peut avoir un effet magique et massif et dans notre cas seulement quelques mois se sont écoulés, des projets peuvent très bien mûrir plusieurs années et émerger quand les conditions seront favorables. 
Ainsi, selon les cas, un stage est en mesure de réactiver les envies de travailler en équipe interdisciplinaire ou au contraire de développer des résistances chez d'autres et c'est seulement si un faisceau de conditions est en place qu'il y aura mobilisation.

Parmi les «bonnes conditions ", il nous apparaît que l'histoire de l'établissement joue un rôle capital, ses habitudes de travail collectif, mais aussi la présence d'une équipe de direction favorisante (soutenant financièrement le projet, valorisant le travail réalisé, « réassurant » les enseignants, prenant en compte les efforts de tous, adultes et élèves). Malgré tout, comme nous le verrons dans notre conclusion, nous pensons que certaines améliorations pourraient être apportées pour augmenter cette efficacité.

Efficience pour l'institution : ces formations sont réputées moins coûteuses que les formations « à l'offre ». Toutefois, il convient de relativiser cette notion, car l'éducation à l'environnement a ses exigences : elle ne peut se dérouler en totalité dans les murs de l'établissement, nécessite forcément des déplacements sur le terrain et l'intervention de partenaires ou intervenants spécialistes qui demandent rémunération. D’autre part, si on comptait le réel investissement des formateurs en temps et en énergie spécifiques à ces stages, les calculs en seraient largement modifiés.

\section{Quelques remarques et propositions...}

48 Ces formations "à la demande» ne peuvent être considérées comme limitées à la stricte durée du temps de stage :

- La rencontre préalable est, nous l'avons constaté dans cette étude, essentielle. Or, actuellement, elle repose sur le strict volontariat des formateurs. Dans leur bonne conscience ils ne pensent pouvoir faire autrement, mais ont des difficultés à faire valider ce temps de travail ou à obtenir des ordres de mission sur les crédits du stage pour se rendre dans l'établissement. Ce n'est actuellement pas officiellement prévu et il conviendrait d'y remédier.

- Un suivi des équipes par les formateurs améliorerait considérablement l'efficacité de ces stages, car une aide a posteriori permettrait d'entretenir les énergies et d'aider à trouver des solutions aux petits obstacles rencontrés par les équipes reprises dans le tourbillon de leur établissement. La notion de suivi-accompagnement est donc à développer (la présence d'un regard extérieur dans un projet a souvent fait ses preuves).

Ces stages « à la demande » sont complémentaires des stages « à l'offre».

- De l'avis des stagiaires qui se sont exprimés il semble important de bien garder ces deux types de formation dans l'Éducation nationale. D'autres études, plus vastes ont été engagées et arrivent à cette même conclusion (voir par exemple «La formation continue des enseignants est-elle utile?", recherche collective aux éditions PUF, octobre 1999).

- De notre côté, en tant que formatrices, nous avons remarqué que l'alternance d'encadrement de ces deux types de stages est particulièrement riche et bénéfique pour nous en nourrissant notre réflexion globale.

L'efficacité des formations à la demande ne pourra être optimale qu'avec une sensibilisation importante des équipes de direction des établissements.

Rappelons aussi que l'éducation à l'environnement est un merveilleux support pour travailler en transversalité (interdisciplinarité, mais aussi participation de toutes les catégories de personnels dans le cadre des projets) et développer la citoyenneté au sein des établissements. Des temps de sensibilisation voire de formation des chefs 
d'établissement seraient à prévoir dans le dispositif d'accompagnement de ces formations.

Nous remercions vivement les collègues stagiaires qui ont accepté de collaborer à cette recherche-action et proposons ces quelques éléments de réflexion, notamment à notre institution.

\section{BIBLIOGRAPHIE}

Bruxelle, Y. (2001). Mieux habiter ensemble la planète. Le partenariat apprenant et sa place dans l'éducation à l'environnement. Mémoire de DURF sous la direction de Paul Taylor, Université François-Rabelais, Tours.

\section{NOTES}

1. Les phrases en italique correspondent à des citations extraites des grilles d'évaluation ou des questionnaires.

\section{AUTEURS}

\section{YANNICK BRUXELLE}

Yannick Bruxelle est professeure agrégée de Sciences de la Vie et de la Terre, en poste au Rectorat de Poitiers notamment pour mettre en place des actions de formation continue visant le développement de projets interdisciplinaires et partenariaux dans le domaine de l'ERE avec des élèves. Engagée pendant trois années dans une recherche avec l'Université de Tours, elle continue cette démarche dans le cadre d'un troisième cycle à l'Université de Rennes 2 (sous la direction de Paul Taylor). Elle est par ailleurs membre active du Réseau national français École et Nature.

\section{DOMINIQUE ELLINGER}

Dominique Ellinger est professeure agrégée de géographie, formatrice à l'IUFM de PoitouCharentes (site de la Vienne), formatrice en éducation à l'environnement dans le cadre d'une équipe partenariale Ifrée-ORE, IUFM, Rectorat, GRAINE lors de stages académiques et nationaux. 